\title{
IS PROCALCITONIN A BETTER DIAGNOSTIC MARKER THAN CRP IN NEONATAL SEPSIS?
}

\author{
Neeraj Kumar¹, R. Dayal², D. Agrawal ${ }^{3}$, P. Kumar', R. Bhatia5 ${ }^{5}$ A. Goyal', D. Verma7
}

\section{HOW TO CITE THIS ARTICLE:}

Neeraj Kumar, R. Dayal, D. Agrawal, P. Kumar, R. Bhatia, A. Goyal, D. Verma. "Is Procalcitonin a better Diagnostic Marker than CRP in Neonatal Sepsis?" Journal of Evolution of Medical and Dental Sciences 2014; Vol. 3, Issue 12, March 24; Page: 3140-3147, DOI: 10.14260/jemds/2014/2257

ABSTRACT: OBJECTIVES: To study the diagnostic value of Procalcitonin (PCT) as compared to Creactive protein (CRP) in the diagnosis of neonatal sepsis. METHODS: This cross sectional study was conducted on 58 neonates in study and 16 in control group admitted in NICU, SN Medical College, Agra. Study group were divided into 2 groups viz. culture positive sepsis and culture negative sepsis. Blood culture, C - reactive protein, Procalcitonin and other investigations for sepsis were performed in all the subjects. RESULTS: PCT levels was raised in significantly higher number of $87.9 \%$ of study cases $(87.9 \%)$ as compared to CRP which was positive in $55.2 \%(\mathrm{p}=0.0002)$. The sensitivity, specificity and positive predictive value (PPV) of PCT in detecting sepsis was $100 \%, 90.09 \%, 100 \%$ respectively as compared to CRP having sensitivity, specificity, PPV as $81.3 \%, 59.1 \%$ and $81.3 \%$ respectively. PCT was a significantly better diagnostic marker in preterm $(\mathrm{p}=0.001)$, low birth weight newborns $(p=0.0002)$ and in early onset sepsis $(p=0.0007)$ in comparison to CRP. CONCLUSION: Serum level of PCT is a more reliable marker than CRP in the diagnosis of neonatal sepsis especially in preterm ( $<37$ wk. gestation), low birth weight $(<2.5 \mathrm{~kg}$ ) newborns and early onset sepsis ( $<72 \mathrm{hrs}$. of life).

KEYWORDS: Neonatal Sepsis, CRP, Procalcitonin.

INTRODUCTION: Neonatal sepsis is a one of the most important cause of morbidity and mortality in newborns throughout the world. In India it contributes to $19 \%$ of all neonatal deaths. ${ }^{1}$ The diagnosis of severe bacterial infection in neonates is often difficult because the clinical signs are non-specific and subtle. Moreover, the laboratory tests routinely used are also inadequate.2,3

In addition to blood culture, other tests that are used for the diagnosis of neonatal sepsis including estimations of the white blood count (WBC), absolute neutrophil count (ANC), micro-ESR and the immature/total neutrophil (I/T) ratio do not have a high sensitivity and specificity in diagnosing neonatal sepsis. ${ }^{4}$ C-reactive protein has been one of the most frequently analyzed parameter for the detection of bacterial infections. Recently, serum procalcitonin (PCT) has been reported as a measurable laboratory marker in the inflammatory response to infection in some studies. ${ }^{5}$ Currently, no single test can be considered as an ideal diagnostic test. So, new laboratory markers for early diagnosis of neonatal sepsis are needed.

Definitive data are lacking in developing countries, regarding PCT as a screening tool in the diagnosis of neonatal sepsis. Hence, this study was undertaken with the aim to determine the diagnostic performance of PCT and also to compare its efficacy with CRP in establishing early diagnosis of neonatal sepsis.

METHODS: This cross-sectional study was conducted on neonates who were admitted to the neonatal intensive care unit (NICU) of pediatrics department, S.N. Medical College, Agra (from March 
2011 to September 2012) with signs and symptoms suggestive of sepsis. This study was approved by the institutional ethical committee and written informed consent was obtained from the parents/guardians.

Inclusion Criteria: Clinically suspected cases of neonatal sepsis with signs and symptoms suggestive of sepsis such as refusal to feed, feeding intolerance, lethargy, excess irritability, high pitched cry, seizures, temperature instability, apnea, respiratory distress, poor perfusion, tachypnea, bradycardia, abdominal distension, necrotizing enterocolitis, vomiting, sclerema were included. For early onset sepsis at least two of the maternal or neonatal risk factors such as fever within 2 weeks prior to delivery, prolonged rupture of amniotic membrane $>24 \mathrm{hr}$., foul smelling and/or meconium stained liquor, single unclean or $>3$ sterile vaginal examinations during labor, prolonged labor (sum of 1 st and $2^{\text {nd }}$ stage of labor $>24 \mathrm{hrs}$.), low birth weight ( $<2500$ grams), prematurity ( $<37$ weeks gestation) and perinatal asphyxia (Apgar score $<4$ at $1 \mathrm{~min}$ ) were also included in the study.

Exclusion Criteria: This group included infants who were already on antibiotics or those who developed the signs of sepsis within 72 hours of discontinuation of the antibiotics and those with congenital anomalies.

Sample Size Estimation: Expected sample size in study and control group was 61 and 13 respectively. It was calculated using Kelsey sample size estimation (Epiinfo-07software) with the assumptions of power $80 \%$, confidence interval $90 \%$ and $25 \%$ expected outcome in control group with control to case ratio of 1 .

The specimens of blood for the sepsis work up were obtained from each neonate prior to the commencement of the antibiotics, which included hematological parameters like total leukocyte count (TLC), absolute neutrophil count (ANC), immature/total neutrophil cell ratio(I/T ratio), microESR, blood culture and sensitivity, CRP and PCT estimation. The samples were processed immediately after collection. Other laboratory tests were done to corroborate the diagnosis of neonatal sepsis wherever clinically indicated to localize the infection such as chest radiograph, lumbar puncture, cultures from body fluids(CSF, urine)etc.

Pathological leukocyte indices were defined according to the criteria of Manroe et al 6 and Lloyd and Oto $^{7}$. TLC $<5000 / \mathrm{mm}^{3}$, ANC $<1800 / \mathrm{mm}^{3}$, I/T ratio $>20 \%$ and micro-ESR $>15 \mathrm{~mm}$ fall were defined as abnormal.

For blood culture, 1-2 ml of blood was added to biphasic media and incubated at $37^{\circ} \mathrm{C}$ for $5-7$ days. Bottles with positive results were sub cultured on Mac Conkey's medium/blood agar/ Chocolate agar every alternate day till 3 subcultures were obtained. The isolated microbes were identified by standard bacteriological methods.

A serum CRP value of $>10 \mathrm{mg} / \mathrm{l}$, as determined by the quantitative rate nephelometry method, was defined as abnormally elevated. ${ }^{8}$

An immunoluminometric assay (LIAISON BRAHMS, Germany) was used for the specific measurement of PCT in serum (detection limit $0.10 \mathrm{ng} / \mathrm{ml}$ ). In this assay, a PCT level of $\geq 0.5 \mathrm{ng} / \mathrm{ml}$ was considered as pathological.

According to clinical signs and symptoms of sepsis and blood culture results, study group was classified into two groups: 
Group A) Culture positive sepsis: positive blood culture with clinical signs and symptoms of sepsis.

Group B) Culture negative sepsis: clinical signs and symptoms present but negative for blood culture.

Control group included healthy neonates with no clinical and laboratory evidence of sepsis.

Statistical Analysis: Data are presented as mean (SD) or number (\%) as appropriate. Categorical variables between groups were analyzed using Fisher's exact test or Chi-square test (as applicable) by epi info 07 software. Diagnostic efficiency was defined as sensitivity, specificity, positive predictive value (PPV) and negative predictive value (NPV). A p value $<0.05$ was considered as significant.

RESULTS: Based on the clinical findings and blood culture results, 58 neonates who were eligible for the study were classified into two groups viz., culture positive sepsis (22 neonates) and culture negative sepsis (36 neonates).

Clinical profile of neonates included in the study is shown in Table I. Out of 58 neonates, $58.6 \%$ were males. There were almost equal no of terms (58.6\%) and preterm infants with majority (69\%) being low birth weight ( $<2500 \mathrm{gms}$ ). $55.3 \%$ cases had early onset sepsis. Overall mean age at the time of presentation was 7.5 \pm 7.67 days and mean age of presentation in early onset of sepsis was $1.68 \pm 0.83$ days. Premature rupture of membrane (PROM) was the most common maternal risk factor identified in 51.6\% (16/31) cases in early onset sepsis followed by multiple PV examination (32.2\%). Most common clinical diagnosis was pneumonia in 33(56.8\%) neonates followed by meningitis in 12 (20.6\%) and urinary tract infection in 5 (8.6\%).

TLC (mean11, 491 $\pm 4,465 / \mathrm{mm} 3$ ) and ANC(mean 7, 317 $\pm 3,896 / \mathrm{mm} 3$ ) was indicative for sepsis in only 3 cases while abnormal I/T ratio(mean $12 \% \pm 0.05$ ) was suggestive in only one case. An elevated micro-ESR was seen in $34.5 \%$ of culture positive cases. Culture was positive in $37.9 \%$ cases.

The commonest organism isolated on blood culture was Staphylococcus aureus $(31.8 \%)$ followed by E. coli (22.7\%). Table II shows CRP to be positive in $55.2 \%$ cases (mean $42.2 \pm 28.5$ $\mathrm{mg} / \mathrm{dl}$ ) and PCT in $87.9 \%$ cases (mean $26.7 \pm 34.9 \mathrm{ng} / \mathrm{dl}$ ). Overall significantly higher proportion of neonates had raised PCT levels $(87.9 \%)(\mathrm{p}=0.002)$ as compared to CRP $(55.2 \%)$. In 22 culture positive cases, 20 subjects had positive PCT values as compared to CRP which was positive in 13 cases $(\mathrm{p}=0.0338)$.

Among 16 controls taken, CRP was positive in 3 cases and PCT was positive in none. Sensitivity of PCT in detecting sepsis was $100 \%$, specificity was $90.9 \%$, PPV was $89.3 \%$ and NPV was $100 \%$. On the other hand, the sensitivity of CRP in predicting sepsis was $81.3 \%$, specificity was $59.1 \%$, PPV was $59.1 \%$ and NPV was $81.3 \%$. The sensitivity of PCT in culture positive cases was $100 \%$, specificity was $91.6 \%$, PPV was $90.9 \%$ and NPV was $100 \%$ whereas for CRP sensitivity, specificity, PPV and NPV in culture positive cases was $81 \%, 67.8 \%, 59 \%$ and $86.3 \%$ respectively.

Variables affecting the positivity of CRP and PCT are given in Table III. Male gender showed significantly higher proportion of PCT positivity $(p=0.0025)$. Preterm infants $(p=0.001)$ and low birth weight newborns ( $p=0.0002)$ also showed PCT rose in significantly larger no of cases than CRP. In cases with early onset sepsis, PCT was raised in 90.3\% cases as opposed to CRP which was raised in $48.39 \%$ cases ( $\mathrm{p}=0.0007$ ).Sensitivity and specificity of CRP was $63.6 \%$ and $80 \%$ as compared to PCT whose sensitivity and specificity was $100 \%$. 
DISCUSSION: Neonatal sepsis with its high mortality rate still remains a diagnostic challenge for the health care providers especially in developing countries. An early and accurate diagnosis of neonatal septicemia helps the clinician in instituting antibiotic therapy at the earliest, thereby reducing the mortality and also helps in avoiding the unnecessary treatment of a non-infected neonate 9.Currently there is no single reliable test for the early definite diagnosis of neonatal sepsis in developing countries, and therefore, there is a need for such diagnostic marker.

In our study sepsis was more common in males (59\%) which is consistent with the findings of Tallur et al. ${ }^{10}$. It may be linked to the x-linked immunoregulatory gene making male neonates more susceptible to infection ${ }^{11}$. This is also probably due to the attitude of the parents who seek medical services more for their male babies than females. Infection was more common in the low birth weight babies (67.8\%) as reported in other studies. ${ }^{12}$ Prematurely born newborns were more commonly infected. This was probably due to their inherent compromised immunity, low complement levels and hypogammaglobulinemia. ${ }^{2}$ Most important maternal risk factor was premature rupture of the membrane (PROM) for early onset sepsis similar to Yancey et al. ${ }^{13}$

In the present study, blood culture was positive in $37.9 \%$ cases which is comparable to study done by Nawshad et al ${ }^{14}$ in which blood culture was positive in $35 \%$ cases. The common organisms isolated were Staphylococcus, E. coli and Klebseilla which was similar to other studies. ${ }^{15,16}$ The readily achievable complete blood count, differential leukocyte count, I/T ratio and micro-ESR although having high specificity in our study, had poor sensitivity for diagnosing sepsis. Gerdes et al ${ }^{17}$ observed in his study that leucocyte indices (TLC, ANC and I/T Ratio) were not accurate enough for early diagnosis of septicemia and their observation was in accordance with our findings.

The C-reactive protein is a classical and sensitive marker of inflammation. ${ }^{18}$. Procalcitonin (PCT) is a 116 amino acid protein, a precursor of calcitonin produced by thyroid. ${ }^{19}$

In many studies sensitivity of PCT in the diagnosis of neonatal sepsis was found to be 83$100 \%$ while the specificity ranged from $70-100 \%{ }^{19,20}$ In our study, sensitivity of PCT was $90.9 \%$ and specificity was $100 \%$ which was found to be superior to CRP having $59.1 \%$ sensitivity and $81.25 \%$ specificity.

In our study PCT was better in diagnosing true positive and true negative cases. PCT with its positive predictive value (PPV) of $100 \%$ and negative predictive value (NPV) of $88.9 \%$ was found to be much superior to CRP having PPV and NPV $81.25 \%$ and 59.1\% respectively. Also, the results of study by Chiesa et al showed that positive and negative predictive values of PCT for neonatal sepsis were $95.3 \%$, and $96.8 \%$ respectively. ${ }^{21}$

On comparing CRP and PCT positivity with various variables, it was observed that male gender, low birth weight ( $<2500$ gms) and prematurity ( $<37$ wks. gestation) showed significantly higher positivity for PCT as compared to CRP. Fendler et al reported PCT to be a better marker than CRP in preterm neonates in diagnosing sepsis. ${ }^{22}$ Auriti et al made observation that PCT was an improved diagnostic marker of sepsis in low birth weight babies as compared to CRP. ${ }^{23}$

PCT positivity for neonates with early onset sepsis was significantly higher as compared to CRP. Among EOS cases, sensitivity and specificity of PCT was 100\% which was higher than CRP having sensitivity and specificity of $63.6 \%$ and $80 \%$ respectively. CRP has been found to be of little value in diagnosing early onset sepsis as the kinetic of CRP is slower than that of PCT, it increases about $6 \mathrm{hrs}$. after onset of inflammation, then doubles every $8 \mathrm{~h}$ and peaks at approximately 36-50 hrs. This is partly due to the fact that CRP lags after the onset of infection because transcription of this 
protein is under the direction of other cytokines. ${ }^{24}$ However, PCT is detectable in the plasma within two hours after the injection of a small amount of bacterial endotoxins, reaches the peak level in 6-8 hours, and reaching a plateau between 12 and 48 hours making it a promising marker for the early identification of infected patients. ${ }^{25}$

Serial monitoring of CRP was not done in this study due to cost restrain which was one of the limitations of the study.

CONCLUSION: The present study showed that PCT is a more reliable marker than CRP and other laboratory parameters in the diagnosis of neonatal sepsis. It has a sensitivity of $100 \%$ and specificity of $90.09 \%$. It is especially more useful in preterm and low birth weight babies in confirming sepsis. In diagnosing early onset sepsis cases it has a sensitivity and specificity as high as $100 \%$ as compared to CRP which had lower $63.6 \%$ sensitivity and $80 \%$ specificity. PCT is an early marker of sepsis, helps in avoiding antibiotic therapy where it is not required and thereby reducing the cost of therapy and also the occurrence of bacterial resistance. Such a benefit might support a wider acceptance of the test in routine practice.

\section{REFERENCES:}

1. Report of the National Neonatal Perinatal Database (National Neonatology Forum) 200203.

2. Rajiv Aggarwal, Nupur Sarkar, Ashok K Deorari, Vinod K. Paul. Sepsis in the newborn. Indian J Pediatr 2001; 68 (12): 1143-1147.

3. Mishra UK, Jacobs SE, Doyle LW, Garland SM. Newer approaches to the diagnosis of early onset neonatal sepsis. Arch Dis Child Fetal Neonatal Ed. 2006; 91(3):208-212.

4. Da Silva 0, Ohlsson A, Kenyon C. Accuracy of leucocyte indices and C-reactive protein for the diagnosis of neonatal sepsis: a critical review. Pediatr Infect Dis J 1995;14: 362-366

5. Carrol ED, Thomson A P, Hart C A. Procalcitonin as a marker of sepsis. Int J Antimicrobe Agents 2002; 20:1-9.

6. Manroe B L, Weinberg A G, Rosenfeld C R, Browne R. The neonatal blood count in health and disease. I. Reference values for neutrophilic cells. J Pediatr1979; 95:89-98.

7. Lloyd BW, Oto A. Normal values for mature and immature neutrophils in very preterm babies. Arch Dis Child 1982; 57:233-235.

8. Mathers NJ, Pohlandt F. Diagnostic audit of C-reactive protein in neonatal infection. Eur J Pediatr1987; 146:147-151.

9. Claudio Chiesa, Alessandra Panero, John F. Osborn, Antonella F. Simonetti, Lucia Pacifico. Diagnosis of Neonatal Sepsis: A Clinical and Laboratory Challenge. Clinical Chemistry 2004; 50: 279-287.

10. Tallur SS, Kasturi AV, Shobha D Nadgir, Krishna BVS. Clinico-bacteriological study of neonatal septicemia in Hubli. Indian J Pediatr 2000; 67(3): 169-174.

11. Waldron I.Sex differences in human mortality: the role of genetic factors. Soc Sci Med.1983; 17(6):321-333.

12. Abida Malik, Shoaib E. Hasani, Harris M. Khan, AzraJ. Ahmed. Nosocomial infections in newborn. Indian Pediatr 2001; 38: 68-71.

13. Yancey MP, Duff P, Kublis P, Clark P, Frentzen BH. Risk factors for neonatal sepsis. ObstetGynecol 1996; 87: 188-194.

14. Nawshad Uddin Ahmed ASM, Azad Chowdhary MAK, Mahbul Hoque, Gary L. Darmstadt. Clinical 
and bacteriological profile of neonatal septicemia in a tertiary level Pediatric Hospital in Bangladesh. Indian Pediatr 2002; 39: 1034-1038.

15. Roy I, Jain A, Kumar M, Agarwal SK. Bacteriology of neonatal septicemia in a Tertiary care Hospital of Northern India. Indian J Med Microbiol 2002; 20(3): 156-159.

16. Karthikeyan G, Premkumar K. Neonatal sepsis: Staphylococcus aureus as the predominant pathogen. Indian J Pediatr2001; 68:715-717.

17. Jeffery S Gerdes, Richard Polin. Early diagnosis and treatment of neonatal sepsis. Indian J Pediatr 1998; 65: 63-71.

18. Chiesa C, Signore F, Assumma M, Buffone E, Tramontezzi P, Osborn JF, et al. Serial measurements of the $C$ reactive protein and interleukin 6 in the immediate postnatal period: the reference intervals and the analysis of the maternal and the perinatal confounders. ClinChem2001; 47:1016-1022.

19. Gendrel D, Raymond J, Coste J. Comparison of procalcitonin with C-reactive protein, interleukin6 and interferon-alpha for differentiation of bacterial vs. viral infections. Pediatr Infect Dis 1999; 18: 875-881.

20. Kafetzis D A, Tigani G S, Costalos C. Immunologic markers in the neonatal period: their diagnostic value and accuracy in infection. Expert Rev MolDign2005; 5:231-239.

21. Chiesa C, Panero A, Rossi N. Reliability of the procalcitonin concentrations in the diagnosis of sepsis in critically ill neonates. Clin Infect Dis 1998;26:664-672

22. Fendler W M, Piotrowski AJ. Procalcitonin in the early diagnosis of nosocomial sepsis in preterm neonates. J Paediatr Child Health.2008; 44(3):114-118.

23. Auriti C, Fiscarelli E, Ronchetti MP, Argentieri M, et al. Procalcitonin in detecting neonatal nosocomial sepsis. Arch Dis Child Fetal Neonatal Ed. 2011.

24. Jaye DL, Waites KB. Clinical applications of C-reactive protein in pediatrics. Pediatr Infect Dis J 1997; 16:735-746.

25. Dandona P, Nix D, Wilson MF, et al. Procalcitonin increase after endotoxin injection in normal subjects. J Clin Endocrinol Metab 1994; 79:1605-1608.

\begin{tabular}{|l|l|c|}
\hline \multirow{2}{*}{ Gender } & Mariables & Frequency (\%) \\
\cline { 2 - 3 } & Female & $34(58.6 \%)$ \\
\hline \multirow{3}{*}{ Birth Weight } & $\geq 2.5 \mathrm{~kg}$ & $24(41.4 \%)$ \\
\cline { 2 - 3 } & $<2.5 \mathrm{~kg}$ & $18(31 \%)$ \\
\hline \multirow{3}{*}{ Gestational Age } & Term & $40(69 \%)$ \\
\hline \multirow{3}{*}{ Materne of onset of sepsis } & Preterm & $34(58.6 \%)$ \\
\cline { 2 - 3 } & EOS & $24(41.4 \%)$ \\
\cline { 2 - 3 } & LOS & $31(55.3 \%)$ \\
\cline { 2 - 3 } & Premature rupture of membrane & $27(46.5 \%)$ \\
\cline { 2 - 3 } & Multiple PV Examination & $16(51.6 \%)$ \\
\cline { 2 - 3 } & Meconium stained liquor & $10(32.2 \%)$ \\
\cline { 2 - 3 } & Prolonged Labor & $6(19.3 \%)$ \\
\cline { 2 - 3 } & Maternal Fever & $4(12.9 \%)$ \\
\cline { 2 - 3 } & Maternal UTI & $3(9.6 \%)$ \\
\hline
\end{tabular}


ORIGINAL ARTICLE

\begin{tabular}{|l|l|c|}
\hline \multirow{4}{*}{$\begin{array}{l}\text { Clinical Presentation } \\
\text { of Study Group }\end{array}$} & Respiratory & $33(56.8 \%)$ \\
\cline { 2 - 3 } & $\begin{array}{l}\text { General } \\
\text { (Refusal to feed, Lethargy) }\end{array}$ & $27(46.5 \%)$ \\
\cline { 2 - 3 } & CNS & $21(36.2 \%)$ \\
\cline { 2 - 3 } & GIT & $16(27.6 \%)$ \\
\cline { 2 - 3 } & CVS & $4(17.2 \%)$ \\
\cline { 2 - 3 } & $\begin{array}{l}\text { Hematological } \\
\text { (bleeding, Jaundice) }\end{array}$ \\
\hline \multirow{2}{*}{ Table 1: Profile of study subjects (n=58) with sepsis } \\
\hline
\end{tabular}

\begin{tabular}{|l|c|c|c|}
\hline \multicolumn{1}{|c|}{ Groups } & $\begin{array}{c}\text { No of cases with } \\
\text { CRP positive (\%) }\end{array}$ & $\begin{array}{c}\text { No of cases with } \\
\text { PCT positive (\%) }\end{array}$ & P value \\
\hline All Cases $(\mathrm{n}=58)$ & $32(55.2)$ & $51(87.9)$ & 0.0002 \\
\hline Culture positive sepsis group $(\mathrm{n}=22)$ & $13(59.1)$ & $20(90.9)$ & 0.0338 \\
\hline Culture negative sepsis group $(\mathrm{n}=36)$ & $19(52.8)$ & $31(86.1)$ & 0.0043 \\
\hline
\end{tabular}

Table 2: Diagnostic performance of CRP and PCT

*p value between CRP and PCT

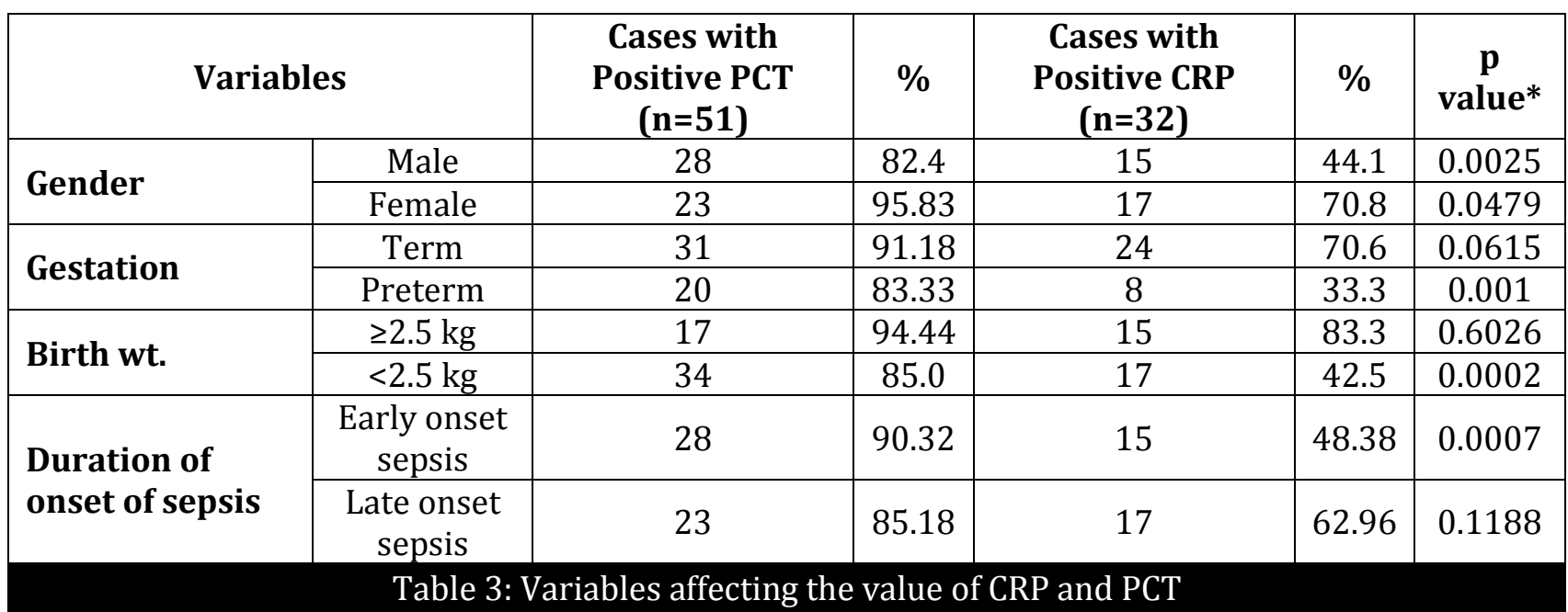

${ }^{*}$ p value between PCT and CRP cases 


\section{ORIGINAL ARTICLE}

\section{AUTHORS:}

1. Neeraj Kumar

2. R. Dayal

3. D. Agrawal

4. P. Kumar

5. R. Bhatia

6. A. Goyal

7. D. Verma

\section{PARTICULARS OF CONTRIBUTORS:}

1. Associate Professor, Department of Paediatrics, S. N Medical College, Agra.

2. Professor, Department of Paediatrics, S. N Medical College, Agra.

3. Assistant Professor, Department of Paediatrics, S. N Medical College, Agra.

4. Assistant Professor, Department of Paediatrics, S. N Medical College, Agra.
5. Assistant Professor, Department of Paediatrics, S. N Medical College, Agra

6. Assistant Professor, Department of Micro Biology, S. N Medical College, Agra.

7. Junior Resident, Department of Paediatrics, S. N Medical College, Agra.

\section{NAME ADDRESS EMAIL ID OF THE CORRESPONDING AUTHOR:}

Dr. Neeraj Kumar,

Warden House,

S. B. H, SNMC, Agra.

E-mail: neerajyadu@rediffmail.com

Date of Submission: 12/02/2014.

Date of Peer Review: 13/02/2014.

Date of Acceptance: 26/02/2014.

Date of Publishing: 22/03/2014. 